\title{
Recent advances in machine intelligence
}

\author{
Suash Deb $^{1} \cdot$ Thomas Hanne $^{2} \cdot$ Simon Fong $^{3}$
}

Published online: 21 July 2016

(C) Springer-Verlag Berlin Heidelberg 2016

Welcome to the special issue (SI) on "Recent Advances in Machine Intelligence". This SI contains a subset of the papers as presented \& appeared in the proceedings of the 1st Intl. Conference on Soft Computing \& Machine Intelligence (ISCMI14), held in New Delhi, India. The papers were shortlisted after a careful scrutiny of the ISCMI14 papers by a group of experts, and the corresponding authors were invited to extend (at least, by $30 \%$ ) their papers and submit for consideration for publication for this SI of SOCO. The submitted papers had undergone the same degree of reviewing, as is applicable for any regular SOCO paper. This rigorous reviewing procedure ultimately resulted in acceptance of only 12 papers. Initially, we planned to provide the SI with uniform representations of papers dealing with Artificial Neural Networks, Fuzzy Logic \& Genetic/Evolutionary Algorithms - the 3 basic components of Machine Intelligence. However, adhering to the strict reviewing protocols of SOCO had made us deviate from this goal. Let us now provide a brief snapshot of the articles included in the SI.

The paper authored by Wang et al. demonstrated how introduction of chaotic theory \& elitism improves the performance of cuckoo search. The one contributed by Mishra

Suash Deb

suashdeb@gmail.com

Thomas Hanne

thomas.hanne@fhnw.ch

Simon Fong

ccfong@umac.mo

1 INNS-India Regional Chapter, Ranchi, India

2 University of Applied Sciences Northwestern Switzerland, Olten, Switzerland

3 University of Macau, Macau, China et al. deals with integer factorization which has immense significance in the field of cryptography \& security. Parkhe et al. had reported a very interesting work on aspect (e.g. direction, screenplay, story, acting)-based analysis of sentiment of a movie review which together influence the opinion of any reviewer. The research effort of Saha et al. demonstrated the utility of semi-supervised clustering over unsupervised or supervised ones for diagnosis of cancer. Affolter et al. introduced a new metaheuristics methodology for addressing index tracking problems with Invasive Weed Optimization. The paper communicated by Bhasin et al. studied diploid genetic algorithms, and the performances were compared with the other algorithms in the context of travelling salesman problem. The manuscript of $\mathrm{Li}$ et al. deals with sentiment analysis for text mining. The contribution of the work reported by Das et al. consists of introducing a new measure of fuzzy information towards determining the criteria of a real-world multi-criteria decision- making problem. The paper of Gee et al. proposed a multi-objective optimization problem for addressing the vehicle routing problem, a very important combinatorial optimization problem. Paramonov et al. investigated the simplification of scoring in the hidden Markov models so as to reduce complexity in speech recognition. Acharya et. al. examined proper partitioning among the clusters as well as formation of appropriate no. of clusters, the two very important aspects of unsupervised classification. The work as reported by Sarvanakumar et al. deals with the control problem of neural networks with distributed time varying delays.

We hope the readers will find the above works informative $\&$ useful for furthering their research works. We would like to take this opportunity of expressing our sincere thanks to the EICs of SOCO, Prof. Antonio DI Nola \& Prof. Vincenzo Loia, for providing us with the opportunity of guest edit- 
ing this SI and for their guidance throughout, which greatly improved the overall quality of the SI. Our appreciation goes to all the reviewers, who despite their busy schedule, extended utmost co operation by sharing their views of the allotted papers on time. We thank all the authors for displaying utmost patience while the process of reviewing was on. Lastly, but certainly not the least, we gratefully acknowledge the help extended by all the editorial staff of SOCO
\& especially to Mr. Anand Venugopal for his dedication \& tremendous support throughout this project.

With kind regards

\section{Compliance with ethical standards}

Conflict of interest All authors declare that they have no conflicts of interest. 